\title{
Rapid response teams and end-of-life care
}

\author{
James Downar MDCM FRCPC
}

\begin{abstract}
Never put off till tomorrow what may be done the day after tomorrow just as well - Mark Twain
\end{abstract}

$\mathrm{R}$ apid response teams (RRTs) were not originally intended to play a role in end-of-life (EoL) care; however, studies from around the world suggest that they do. In the current issue of the Canadian Respiratory Journal, Tam et al (1) (pages 302-307) present the results of a two-year retrospective chart review showing that an RRT participated in EoL discussions in 319 cases. They found that RRT participation in EoL discussions often triggered a change in resuscitation orders and was associated with a lower likelihood of intensive care unit (ICU) admission and a higher likelihood of palliative care consultation.

These findings add to a growing body of evidence for the important role that RRTs play in EoL care. Overall, approximately one in 10 new RRT consultations result in a limitation of treatment order, and RRTs are consulted to see $25 \%$ to $50 \%$ of patients who die in hospital (2-4). In particular, RRTs appear to play an important role in EoL conversations that occur later in hospitalization and after hours (5). Of note, the incidence of RRT involvement in EoL discussions is broadly consistent among studies from around the world, suggesting that this is not an idiosyncratic cultural phenomenon, but rather a reflection of human nature.

Humans tend to procrastinate, and EoL discussions are no exception. Studies routinely show that patients want to talk about their EoL preferences, and physicians believe that EoL discussions are important. Yet, EoL discussions are typically delayed until very late in the course of disease, and are often prompted by a significant event such as an unscheduled hospital admission or a clinical deterioration. For many patients, families and physicians, EoL conversations cannot occur unless they are in earnest. The importance of the RRT then becomes obvious - it is the first team to arrive when a clinical deterioration appears to be imminent, and it is the final opportunity to deviate from the 'default' pathway of aggressive care before the patient is admitted to the ICU.

Is it appropriate for the RRT to be discussing EoL care? In many ways, the RRT is well suited to this role. RRT staff have experience with critical care and are the most appropriate individuals to obtain 'consent' for a course of ICU treatment. They also have experience with this type of discussion from their time in the ICU. Some may argue that it is the role of the most responsible physician(s) (MRPs) to discuss resuscitation orders and EoL care. However, in that sense, EoL discussions are no different than fluid boluses, diuretics, antibiotics and many of the other common RRT interventions. In theory, the MRPs should be the ones using these interventions in a timely fashion to prevent deterioration. The RRT exists because they don't.

There are also concerns about using the RRT in this manner. The RRT usually has no previous relationship with the patient or family; therefore, the emotional and time burden of taking on this role for a large proportion of inpatients could be overwhelming. The RRT may also be forced to make decisions in a crisis situation, without the benefit of input from the patient (who is clinically deteriorating), which is not ideal. Furthermore, the RRT's behaviour may be influenced by the availability of ICU resources. Stelfox et al (6) found that the availability or unavailability of ICU beds was significantly associated with the probability that an RRT consult would result in an ICU admission or a limitation of treatment order. However, the overall hospital mortality for these patients was not affected by bed availability, suggesting that empty beds were leading to 'soft' admissions, rather than full beds leading to inadequate care. Following on the earlier theme, an empty ICU bed was simply another opportunity to delay the EoL conversation.

Although we may wish to move EoL discussions to earlier in the disease course, RRTs will always have a role to play in EoL discussions and care, and we need to equip them properly for this role. Communication training is a critical element, and can improve both comfort and skill at participating in challenging discussions. But highquality EoL care is more complicated than simply avoiding an ICU admission. We found that RRTs often missed important opportunities to improve symptom management and psychosocial issues (4); therefore, we should consider automatic consultations to palliative care and other services, as well as preprinted order sets for comfort care. Tam et al (1) found that the introduction of a preprinted order set was associated with an increase in limitation of treatment orders; however, improving symptom control and other aspects of EoL care may be more challenging. If a complex, well-funded, multicomponent EoL intervention is ineffective for improving EoL care in the ICU (7), then we must temper our expectations for a similar intervention on the wards.

\section{REFERENCES}

1. Tam B, Salib M, Fox-Robichaud A. The effect of rapid response teams on end-of-life care: A retrospective chart review. Can Respir J 2014;21:302-7.

2. Jones D, Moran J, Winters B, Welch J. The rapid response system and end-of-life care. Curr Opin Crit Care 2013;19:616-23.

3. Downar J, Barua R, Rodin D, et al. Changes in end of life care 5 years after the introduction of a rapid response team:

A multicentre retrospective study. Resuscitation 2013;84:1339-44.

4. Downar J, Rodin D, Barua R, et al. Rapid response teams, do not resuscitate orders, and potential opportunities to improve end-oflife care: A multicentre retrospective study. J Crit Care 2013;28:498-503.

5. Jones DA, Bagshaw SM, Barrett J, et al. The role of the medical emergency team in end-of-life care: A multicenter, prospective, observational study. Crit Care Med 2012;40:98-103.

6. Stelfox HT, Hemmelgarn BR, Bagshaw SM, et al. Intensive care unit bed availability and outcomes for hospitalized patients with sudden clinical deterioration. Arch Intern Med 2012;172:467-74.

7. Curtis JR, Nielsen EL, Treece PD, et al. Effect of a qualityimprovement intervention on end-of-life care in the intensive care unit: A randomized trial. Am J Respir Crit Care Med 2011;183:348-55. 


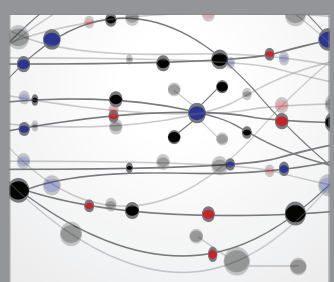

The Scientific World Journal
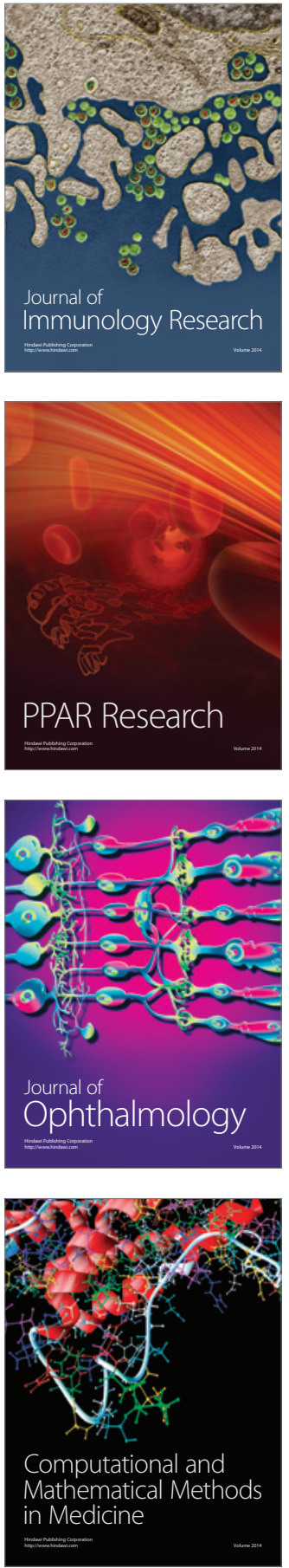

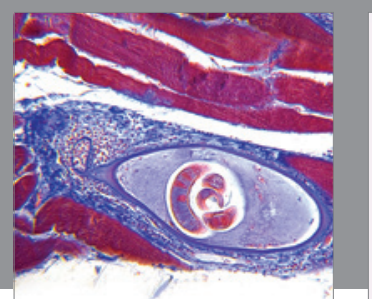

Gastroenterology Research and Practice

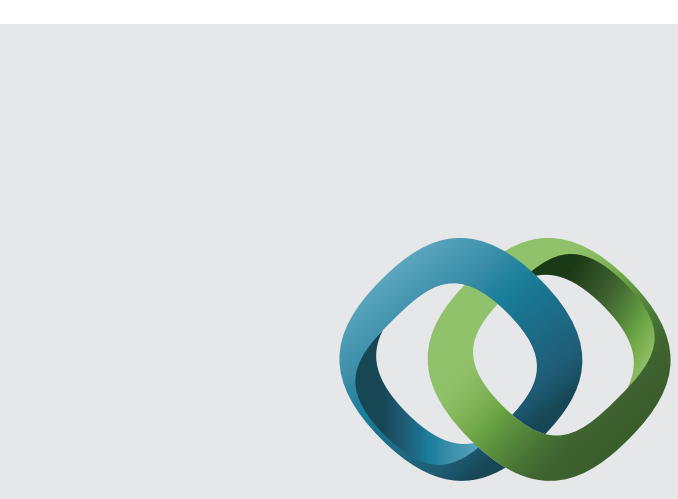

\section{Hindawi}

Submit your manuscripts at

http://www.hindawi.com
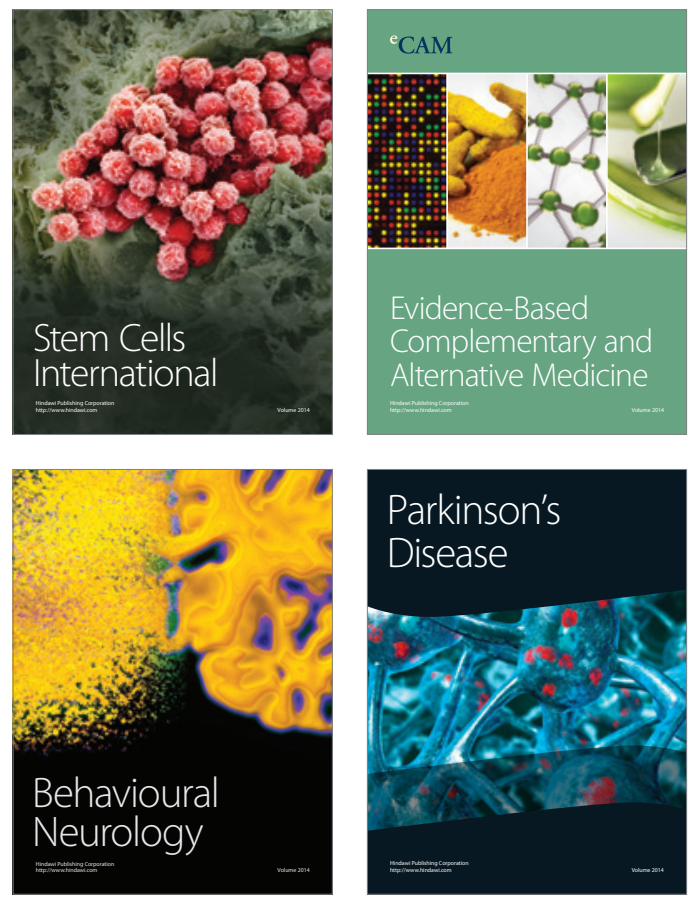
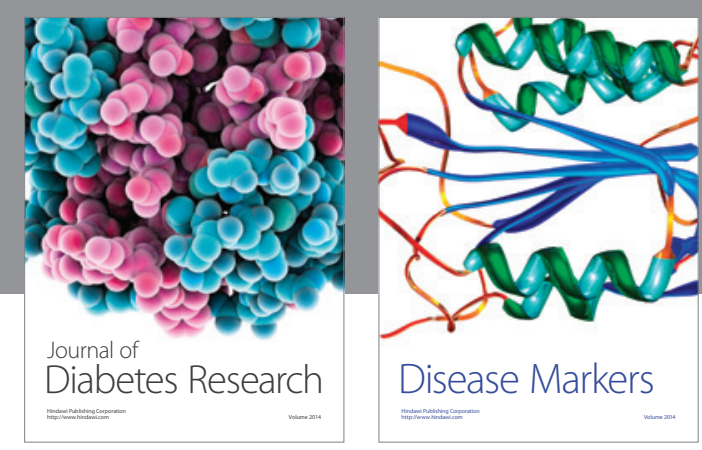

Disease Markers
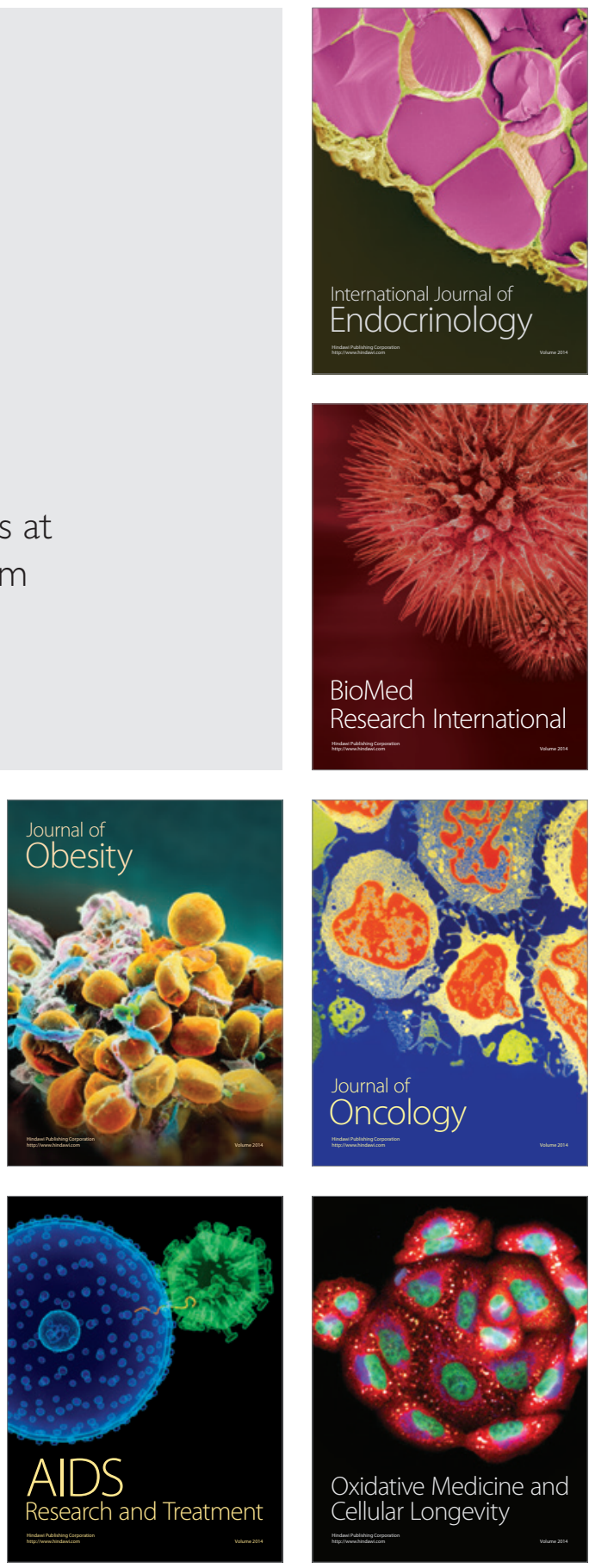